\title{
Photocatalytic applications with CdS•block copolymer/exfoliated graphene nanoensembles: Hydrogen generation and degradation of Rhodamine B
}

\author{
T Skaltsas, N Karousis, S Pispas and N Tagmatarchis* \\ Theoretical and Physical Chemistry Institute, National Hellenic Research \\ Foundation, 48 Vassileos Constantinou Avenue, 11635 Athens, Greece \\ Email: tagmatar@eie.gr
}

\begin{abstract}
Amphiphilic block copolymer poly(isoprene- $b$-acrylic acid) (PI- $b$-PAA) stabilized exfoliated graphene in water and allowed the immobilization of semiconductor CdS nanoparticles forming CdS•PI- $b$-PAA/graphene. Characterization with HR-TEM and EDX justified the success of preparation and revealed the presence of spherical CdS. Moreover, UV-Vis and photoluminescence assays suggested that electronic interactions within CdS•PI- $b$-PAA/graphene exist as evidenced by the significant quenching of the characteristic emission of CdS by exfoliated graphene. Photoillumination of CdS•PI- $b$-PAA/graphene, in the presence of ammonium formate as quencher for the photogenerated holes, resulted on the generation of hydrogen by water splitting, monitored by the

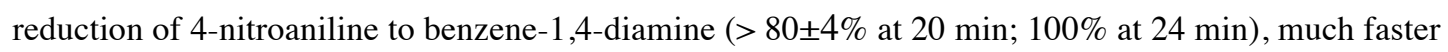
and efficient as compared when reference CdS•PI- $b$-PAA was used as photocatalyst $(<30 \pm 3 \%$ at 20 $\mathrm{min} ; 100 \%$ at $240 \mathrm{~min}$ ). Moreover, Rhodamine B was photocatalytically degraded by CdS $\bullet \mathrm{PI}-b$ PAA/graphene, with fast kinetics under visible light illumination in the presence of air. The enhancement of both photocatalytic processes by CdS $\bullet \mathrm{PI}-b$-PAA/graphene was rationalized in terms of effective separation of holes-electrons, contrary to reference CdS•PI- $b$-PAA, in which rapid recombination of the hole-electron pair is inevitable due to the absence of exfoliated graphene as suitable electron acceptor.
\end{abstract}




\section{Introduction}

Semiconductors due to their unique electronic structure have the ability to promote photo-induced catalytic phenomena. In this context, photogenerated holes and electrons, as derived upon migration of an electron from the valence to the conduction band of the semiconductor, play an important role in photocatalysis. However, rapid charge-recombination, resulting in low photocatalytic efficiency and thus limiting practical applicability, is a major drawback for those systems. To overcome this obstacle, suppression of the charge-recombination rate is a key-issue which can be addressed by considering donoracceptor hybrid nanostructures in which semiconductors are combined with electron-acceptors such as graphene which, due to its superior electron mobility and high specific surface area, enhances chargeseparation via electron-transfer processes. Recently, the combination of CdTe quantum dots with graphene was reported and photoinduced electron-transfer phenomena from CdTe to graphene were found $[1,2]$. Moreover, numerous studies dealt with the combination of a variety of semiconductor quantum dots and graphene oxide $(\mathrm{GO})$ or reduced graphene oxide (RGO) toward the preparation of photocatalytic systems for hydrogen generation and degradation of pollutants [3-6]. However, since GO possesses disrupted electronic network deficient of novel electronic properties, new routes for obtaining graphene sheets that preserve to large extent the high conductivity of the material are required. Meanwhile, reduction of GO partially occurs, hence, hampering the complete restoration of the $\mathrm{sp}^{2}$ network and resulting to material with properties that significantly deviate from those of pristine graphene. For the aforementioned reasons, both GO and RGO are rather unappealing for technological applications in which high and efficient charge transport is required, particularly those in photocatalysis, regardless that GO and/or RGO have already been combined with semiconductor quantum dots [7-14].The alternative route for achieving high-quality graphene sheets, yet well-dispersed in organic solvents, is by exfoliating graphite [15-17]. Without a doubt, liquid exfoliation of graphite results on the acquisition of large quantities of high quality graphene, which in turn can further boost its direct applicability. In this context, it is absolutely timely and significantly important to integrate semiconducting quantum dots onto liquid exfoliated graphene, by non-covalent means, aiming to keep intact the $\pi$-electronic network of graphene, and investigate the efficiency of the hybrid material in photocatalytic applications, namely, to generate hydrogen and degrade organic dyes upon photoirradiation.

Photocatalytic water splitting is a promising, clean, environmental friendly and economic approach to produce hydrogen by solar energy [18,19]. Besides, since photocatalytic water splitting into stoichiometric $\mathrm{H}_{2}$ and $\mathrm{O}_{2}$ is regarded as artificial photosynthesis, electron donor-acceptor ensembles composed of semiconductor CdS and exfoliated graphene can be of special interest. In addition, environmental protection from noxious waste is a major issue and advanced oxidative processes for the 
destruction of synthetic organic species resistant to conventional methods are needed [20-23]. Such oxidative processes rely on in-situ generation of highly reactive radical species, by using solar energy, in the presence of accumulated electrons (i.e. by reduction of $\mathrm{O}_{2}$ ), those for example that are yielded in donor-acceptor ensembles upon electron-transfer phenomena at the acceptor site. Herein, aqueous ensembles of semiconductor CdS nanoparticles combined with block copolymer stabilized exfoliated graphene were tested for the first time in photocatalysis. Particularly, generation of hydrogen by water splitting as observed by monitoring the reduction of 4-nitroaniline to the corresponding benzene-1,4diamine, upon photoillumination of $\mathrm{CdS} /$ graphene ensembles in the presence of ammonium formate as quencher for the photogenerated holes was achieved. Moreover, degradation of Rhodamine $\mathrm{B}(\mathrm{RhB})$, one of the most important and common xanthene cationic dyes [24-26], with fast kinetics under visible light illumination of $\mathrm{CdS} /$ graphene in the presence of air was attained. Both photocatalytic processes were due to electron-transfer phenomena that take place within the $\mathrm{CdS} /$ graphene nanoensemble.

\section{Methods}

\subsection{Materials and Methods}

All reagents and solvents were purchased from Aldrich and used without further purification. Graphene was produced after liquid exfoliation of graphite flakes in $N$-methylpyrrolidone (NMP) [27]. Poly(isoprene- $b$-acrylic acid), abbreviated as PI- $b$-PAA $\left(\mathrm{M}_{\mathrm{w}}=42,500, \mathrm{M}_{\mathrm{w}} / \mathrm{M}_{\mathrm{n}}=1.16,10 \mathrm{wt} \%\right.$ PI) was synthesized following to previously reported procedures [28]. Steady state UV-Vis electronic absorption spectra were recorded on a Perkin-Elmer (Lambda 19) UV-Vis-NIR spectrophotometer. Steady state emission spectra were recorded on a Fluorolog-3 Jobin Yvon-Spex spectrofluorometer (model GL3-21). HR-TEM measurements were carried out using a JEM-2100F (JEOL) high-resolution field-emission gun TEM operated at $80 \mathrm{keV}$ at room temperature and under a pressure of $10^{-6} \mathrm{~Pa}$. HR-TEM images were recorded with a charge-coupled device with an exposure time of typically 1s. Energy dispersive X-ray (EDX) spectroscopy measurements were performed using the same microscope equipped with a super atmospheric thin-window X-ray detector.

\subsection{Preparation of graphene/PI- $b-P A A$}

A solution of PI- $b$-PAA in NMP $(2 \mathrm{mg} / \mathrm{mL})$ was added to a dispersion of exfoliated graphene in NMP $(24.4 \mu \mathrm{g}$ in $2 \mathrm{~mL})$ and stirred gently overnight at room temperature. 


\subsection{Preparation of $C d S \cdot P I-b-P A A$}

An aqueous solution $(2 \mathrm{~mL})$ of $\mathrm{CdCl}_{2} \mathrm{O}_{8}(5.6 \mathrm{mg} / \mathrm{mL})$ was added to a solution $(1 \mathrm{~mL})$ of PI- $b$-PAA in NMP (2 mg/mL) and left stirring overnight at room temperature. Subsequently, an aqueous solution (2 $\mathrm{mL})$ of $\mathrm{Na}_{2} \mathrm{~S}(6.4 \mathrm{mg} / \mathrm{mL})$ was added and the mixture stirred for $1 \mathrm{~h}$ at room temperature.

\subsection{Preparation of $C d S \bullet P I-b-P A A / g r a p h e n e ~ e n s e m b l e s$}

An aqueous solution $(2 \mathrm{~mL})$ of $\mathrm{CdCl}_{2} \mathrm{O}_{8}(5.6 \mathrm{mg} / \mathrm{mL})$ was added in graphene/PI- $b$-PAA dispersion in NMP (3 mL) and stirred overnight at room temperature. Then, an aqueous solution $(2 \mathrm{~mL})$ of $\mathrm{Na}_{2} \mathrm{~S}(6.4$ $\mathrm{mg} / \mathrm{mL}$ ) was added and the mixture stirred for $1 \mathrm{~h}$ at room temperature.

\subsection{Photocatalytic $\mathrm{H}_{2}$ evolution}

The photocatalytic $\mathrm{H}_{2}$ evolution was carried out in a special Pyrex vessel sealed with a silicone rubber septum, at room temperature under nitrogen atmosphere. The Pyrex vessel was positioned inside a cylindrical vessel surrounded by a circulating $\mathrm{NaNO}_{2} 1 \mathrm{M}$ aqueous solution as UV-cut-off filter, thus ensuring that illumination was only by visible light. The light source used was a $500 \mathrm{~W}$ Xenon lamp, which was positioned $20 \mathrm{~cm}$ away from the reactor. Typically, CdS•PI- $b$-PAA/graphene as photocatalyst (1.5 mL of a $5.57 \mathrm{mg} / \mathrm{mL}$ solution) was added to an aqueous solution of 4-nitroaniline ( $2 \mathrm{~mL}$ of a 10 $\mathrm{mg} / \mathrm{L}$ aqueous solution) and ammonium formate $(2 \mathrm{mg})$, which was used as sacrificial agent. Prior to visible light irradiation, the reaction mixture was bubbled with nitrogen for $30 \mathrm{~min}$ to remove the dissolved oxygen. At given irradiation time intervals, $3 \mathrm{~mL}$ of the reaction mixture was sampled and separated by centrifugation (4.200 rpm, $5 \mathrm{~min}$.). The supernatant was analysed by UV-Vis spectroscopy and the concentration of 4-nitroaniline was determined by monitoring changes in the absorption band centred at $380 \mathrm{~nm}$.

\subsection{Photocatalytic degradation of $R h B$}

The degradation of aqueous RhB was carried out in a special Pyrex vessel, which was positioned inside a cylindrical vessel surrounded by a circulating $\mathrm{NaNO}_{2} 1 \mathrm{M}$ aqueous solution as UV-cut-off filter, thus ensuring that illumination was only by visible light. The light source used was a $500 \mathrm{~W}$ Xenon lamp, which was positioned $20 \mathrm{~cm}$ away from the reactor. The CdS•PI- $b$-PAA/graphene photocatalyst $(13 \mathrm{mg}$, $3.85 \mu \mathrm{mol} / \mathrm{mg})$ was added in an aqueous solution of $\mathrm{RhB}\left(2.5 \mathrm{~mL}, 10^{-5} \mathrm{M}\right)$ and the mixture stirred for $18 \mathrm{~h}$ in the dark in order to achieve a suitable adsorption-desorption equilibrium of the dye on the surface of 
the catalyst. The experiment took place under continuous air flow. At given irradiation time intervals, 3 $\mathrm{mL}$ of the reaction mixture was sampled and separated by centrifugation (4.2 rpm, $5 \mathrm{~min}$.). The concentration of RhB was determined by monitoring changes in the absorption band centred at $555 \mathrm{~nm}$.

\section{Results and Discussion}

\subsection{Synthesis and characterization of CdS•PI-b-PAA/graphene}

In line with our previously described methodology [27], exfoliated graphene obtained upon tip sonication of a graphite suspension in NMP was further treated with the amphiphilic block copolymer PI- $b$-PAA in order to achieve long period stabilization in aqueous media [29]. Subsequently, the carboxyl groups of the poly(acrylic acid) block of the copolymer in the PI- $b$-PAA/graphene hybrid material were used as anchors for the capture of $\mathrm{Cd}^{2+}$ cations, which after gentle stirring with $\mathrm{Na}_{2} \mathrm{~S}$ for $1 \mathrm{~h}$ were successively converted to $\mathrm{CdS}$ nanoparticles, thus yielding the CdS•PI- $b$-PAA/graphene ensemble.

The aforementioned $\mathrm{CdS} \bullet \mathrm{PI}-b$-PAA/graphene ensemble was characterized in full by complementary microscopic and spectroscopic means. High-resolution transmission electron microscopy (HR-TEM) images of $\mathrm{CdS} \bullet \mathrm{PI}-b$-PAA/graphene clearly indicated the presence of round-shaped monodispersed nanoparticles of average diameter $3-5 \mathrm{~nm}$, spread all over the exfoliated graphene sheets with higher density at graphene wrinkled edges (Figure 1 (a)). In some cases, TEM imaging of monolayered graphene in CdS•PI- $b$-PAA/graphene ensemble was possible, identified from selected area electron diffraction (SAED) and fast Fourier transform (FFT) in the selected areas (see inset of Figure 1 (a)). Energy dispersive X-ray (EDX) spectroscopy confirmed the presence of $\mathrm{Cd}$ and $\mathrm{S}$ in the sample (peaks at $3.1 \mathrm{keV}$ and $2.3 \mathrm{keV}$, respectively) in a 1:1 ratio (Figure $1(\mathrm{~b})$ ), indicating that the spherical particles observed in HR-TEM images represent CdS nanoparticles. At the same time, reference images of CdS nanoparticles stabilized in the presence of PI- $b$-PAA, prepared under similar experimental conditions but in the absence of exfoliated graphene, revealed the same spherical morphology with diameter in the range of 3-5 nm (Figure 1 (c)), while EDX spectroscopy further proved the presence of $\mathrm{Cd}$ and $\mathrm{S}$ in the reference CdS•PI$b$-PAA material (Figure $1(\mathrm{~d})$ ). 

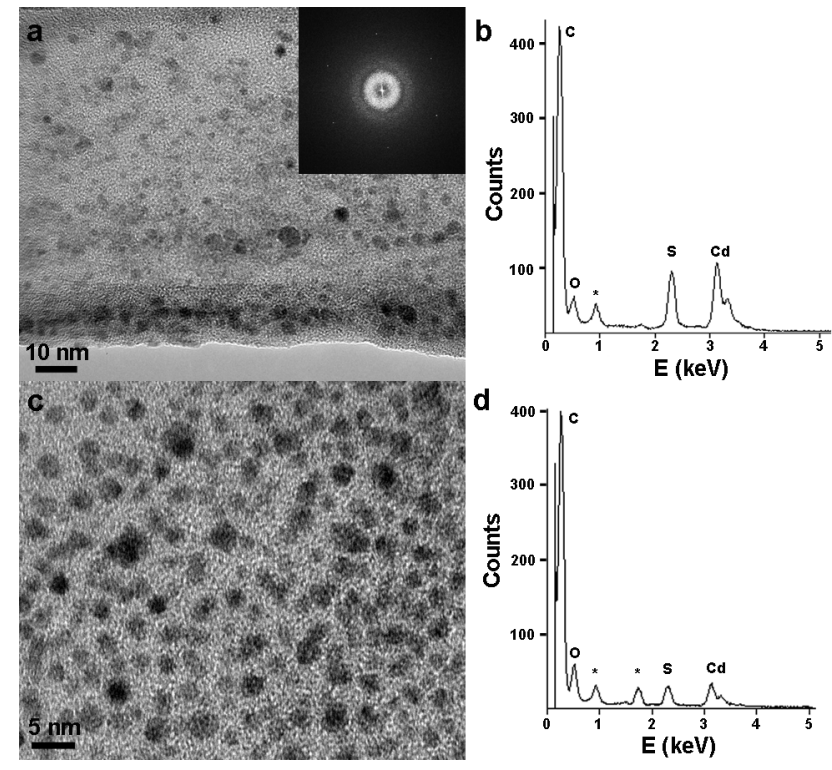

Figure 1. HR-TEM images [(a), (c)] and EDX spectra [(b), (d)] of CdS•PI- $b$-PAA/graphene ensemble and reference CdS•PI- $b$-PAA material, respectively. In the EDX spectra [(b), (d)], stars denote the elements $\mathrm{Cu}$ and $\mathrm{Si}$, which are detected because of their presence in the microscope equipment, sample holder, and crystal detector. The inset in HR-TEM image of CdS•PI- $b$-PAA/graphene ensemble shows the FFT from the specific selected area electron diffraction.

The CdS•PI- $b$-PAA and CdS•PI- $b$-PAA/graphene ensembles form yellow and yellow-grey solutions in a mixture of NMP: $\mathrm{H}_{2} \mathrm{O}(1: 1)$, respectively, and were stable for several weeks, without observing any precipitation, allowing their spectroscopic study in solution. In this context, the UV-Vis spectrum of $\mathrm{CdS} \bullet \mathrm{PI}-b$-PAA revealed an absorption band at $430 \mathrm{~nm}$ (Figure 2 (a)), characteristic of the electronic absorption of CdS, however, considerably blue shifted as compared to the absorption band of bulk CdS centered at $\sim 512 \mathrm{~nm}$, indicative of the quantum size effect. Furthermore, the absorption spectrum of $\mathrm{CdS} \bullet \mathrm{PI}-b$-PAA/graphene showed a continuing absorption due to the presence of exfoliated graphene sheets, with an evolving band at around $440 \mathrm{~nm}$, suggesting firstly the efficient formation of the CdS•PI$b$-PAA/graphene nanoensemble and secondly that the size of CdS in CdS•PI- $b$-PAA/graphene is similar to the one in the reference material CdS•PI- $b$-PAA. The latter observation is in full accordance with the HR-TEM images described earlier (cf. Figure 1 (a) and (c)). Besides that, photoluminescence assays revealed the presence of strong electronic interactions between CdS and exfoliated graphene sheets in the aqueous CdS•PI- $b$-PAA/graphene ensemble. The characteristic strong emission of CdS in CdS•PI- $b$-PAA at $649 \mathrm{~nm}$, as observed upon photoexcitation at $400 \mathrm{~nm}$, was found significantly quenched and red-shifted by $19 \mathrm{~nm}$ in the CdS•PI- $b$-PAA/graphene ensemble (Figure 2 (b)). The latter observation manifests strong electronic interactions in the excited states between CdS and exfoliated graphene and further suggests that 
photogenerated electrons migrate and transport from the excited state of $\mathrm{CdS}$ in exfoliated graphene sheets, within the $\mathrm{CdS} \bullet \mathrm{PI}-b$-PAA/graphene nanoensemble.
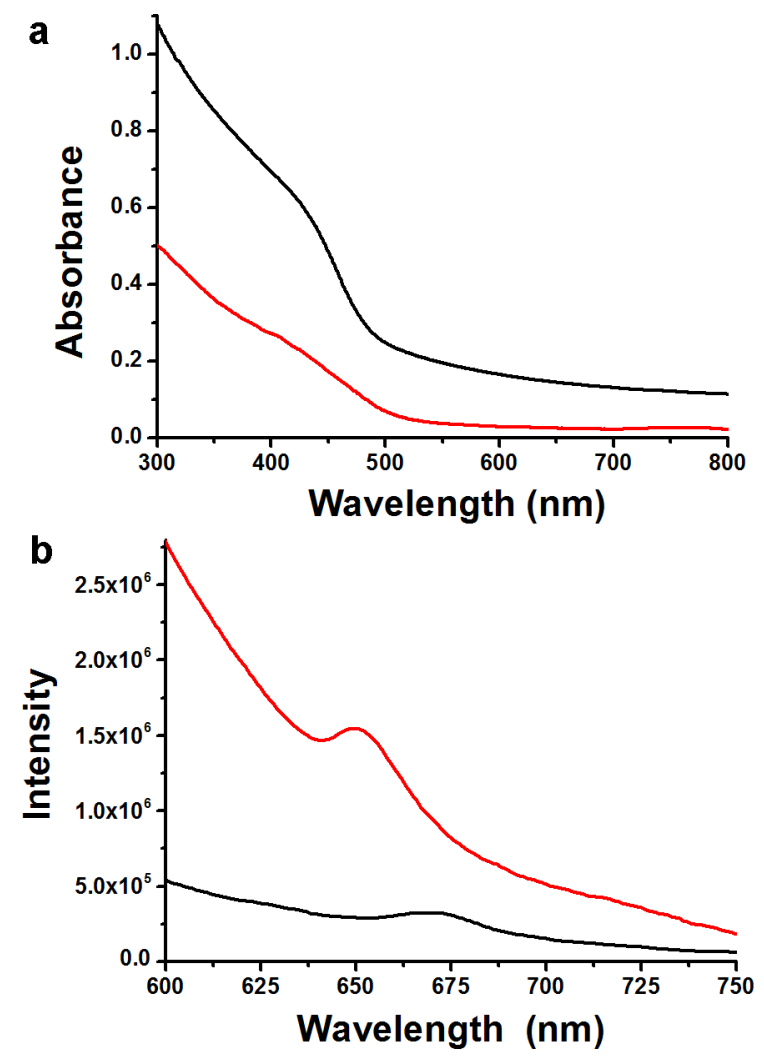

Figure 2. UV-Vis absorption (a) and photoluminescence (b) spectra of CdS•PI- $b$-PAA/graphene nanoensemble (black) and reference CdS•PI- $b$-PAA (red), respectively, obtained in NMP: $\mathrm{H}_{2} \mathrm{O}(1: 1)$. The excitation wavelength was $400 \mathrm{~nm}$, and the concentrations were adjusted so that CdS in the two samples exhibited equal absorbance at the excitation wavelength.

\subsection{Photocatalytic $\mathrm{H}_{2}$ evolution}

Having comprehensively characterized the CdS•PI- $b$-PAA/graphene ensemble, its photocatalytic activity toward producing hydrogen by water splitting under visible light irradiation was evaluated. To this end, 4nitroaniline was reduced to benzene-1,4-diamine upon visible light illumination, under continuous $\mathrm{N}_{2}$ purge, in the presence of $\mathrm{CdS} \bullet \mathrm{PI}-b$-PAA/graphene and ammonium formate as quencher for the photogenerated holes. The photocatalytic activity of CdS•PI- $b$-PAA/graphene on producing $\mathrm{H}_{2}$ was evaluated by monitoring temporal changes in the absorption spectrum of 4-nitroaniline under visible light 
irradiation. Figure 3 (a) shows that the characteristic absorption band at $386 \mathrm{~nm}$ attributed to 4 nitroaniline gradually decreased and disappeared after $24 \mathrm{~min}$. in the presence of CdS•PI-bPAA/graphene ensemble as photocatalyst, while at the same time two new absorption bands evolved, at 323 and $264 \mathrm{~nm}$, indicating the formation of the reduced product. Notably, the generation of $\mathrm{H}_{2}$ as identified by the formation of benzene-1,4-diamine was significantly slower when reference CdS•PI- $b$ PAA was used as photocatalyst (Figure 3 (b)), with the transformation completed after 240 min. Following the temporal course of the 4-nitroaniline reduction (Figure 3 (c)), it is obvious that within 20 min. more than $80 \pm 4 \%$ of 4 -nitroaniline was reduced by the CdS $\bullet P I-b$-PAA/graphene ensemble, while after the same period of time less than $30 \pm 3 \%$ was reduced by the reference CdS•PI- $b$-PAA material. The latter result highlights the high efficiency of $\mathrm{CdS} \bullet \mathrm{PI}-b$-PAA/graphene as photocatalyst for hydrogen production in comparison to reference $\mathrm{CdS} \bullet \mathrm{PI}-b$-PAA. Moreover, control experiments performed in dark or with only exfoliated graphene (i.e. without $\mathrm{CdS}$ ) were negative (i.e. no changes were observed in the UV-Vis spectrum of 4-nitroaniline after $4 \mathrm{~h}$ of visible light irradiation). In other words, those control experiments showed the absence of any hydrogen evolution as evidenced by the stability of 4-nitroaniline. Collectively, this set of experiments showcasing the hydrogen generation by water splitting upon lightirradiation of $\mathrm{CdS} \bullet \mathrm{PI}-b$-PAA/graphene, clearly indicates the important role of exfoliated graphene as electron-acceptor in the CdS•PI- $b$-PAA/graphene ensemble, in which the photoinduced electron-transfer phenomena governing the nanoensemble decelerate the recombination rate of the electron-hole pair, contrary to the case of CdS•PI- $b$-PAA in which ultra-fast recombination occurs.
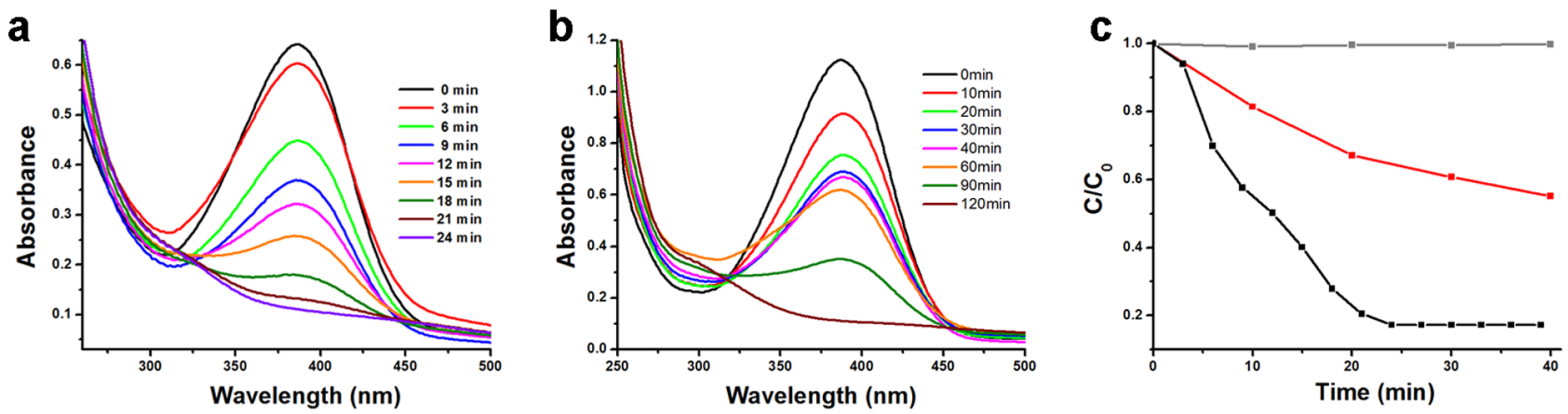

Figure 3.UV-Vis absorption spectra of 4-nitroaniline over (a) CdS•PI- $b$-PAA/graphene, and (b) CdS•PI$b$-PAA, under visible light irradiation, in the presence of ammonium formate as sacrificial agent and $\mathrm{N}_{2}$ purge. (c) Photocatalytic performance of CdS $\bullet P I-b$-PAA/graphene (black), reference CdS $\bullet P I-b$-PAA (red) and exfoliated graphene (grey) in the reduction of aqueous 4-nitroaniline under visible light irradiation in the presence of $\mathrm{N}_{2}$. 


\subsection{Photocatalytic degradation of $R h B$}

The photocatalytic activity of nanostructured $\mathrm{CdS} \bullet \mathrm{PI}-b$-PAA/graphene was further investigated by monitoring its influence on the degradation of RhB. Briefly, CdS•PI- $b$-PAA/graphene was initially added to aqueous $\mathrm{RhB}$ and the reaction mixture protected from light was kept under stirring overnight to establish the appropriate adsorption/desorption equilibrium. Moreover, the photocatalytic degradation experiments took place under continuous airflow ensuring the presence of excess $\mathrm{O}_{2}$ in the reaction mixture. Although a strong band centered at $555 \mathrm{~nm}$ characterizes the absorption spectrum of RhB, UVVis spectral changes were developed when aqueous $\mathrm{RhB}$ was irradiated by visible light in the presence of air and catalytic amounts of CdS•PI- $b$-PAA/graphene. Evidently, the absorption band of RhB was gradually reduced as a function of elapsing time while simultaneously was blue-shifted by $16 \mathrm{~nm}$ (i.e. absorption centered at $539 \mathrm{~nm}$ ) after $90 \mathrm{~min}$ and eventually by $44 \mathrm{~nm}$ (i.e. absorption centered at $511 \mathrm{~nm}$ ) after 150 min (Figure 4 (a)). Such spectral changes in the electronic absorption profile of RhB correspond to the loss of one and three $N$-ethyl groups and the formation of $N, N, N^{\prime}$-triethylrhodamine and $N$-ethylrhodamine, respectively, according to previous reports [30]. In stark contrast, aqueous RhB was found photostable in the absence of CdS•PI- $b$-PAA/graphene (i.e. no changes were observed in the UV-Vis spectrum after $3 \mathrm{~h}$ of visible light irradiation). Furthermore, no changes on the electronic absorption spectrum of $\mathrm{RhB}$ were observed in the presence of $\mathrm{CdS} \bullet \mathrm{PI}-b$-PAA/graphene but without photoirradiation, thus suggesting that visible light illumination is a key-factor for the degradation process. Additional reference experiments were also performed with exfoliated graphene (i.e. without $\mathrm{CdS}$ ) or CdS•PI- $b$-PAA (i.e. without graphene) and found that photoirradiated $\mathrm{RhB}$ remained intact in the presence of exfoliated graphene, while partially transformed, however, with rather slow kinetics, by CdS•PI- $b$-PAA (Figure 4 (b)). The normalized temporal concentration changes $\left(\mathrm{C} / \mathrm{C}_{0}\right)$ of $\mathrm{RhB}$ during the photocatalytic process (Figure 4 (c)), clearly illustrate that CdS•PI-b-PAA/graphene exhibit superior photocatalytic performance than CdS•PI- $b$-PAA. Evidently, more than $90 \pm 2 \%$ of RhB was decomposed after 150 min of visible light irradiation by $\mathrm{CdS} \bullet \mathrm{PI}-b$-PAA/graphene, whereas the degradation percentage drops to less than $45 \pm 5 \%$ in the presence of CdS•PI- $b$-PAA.
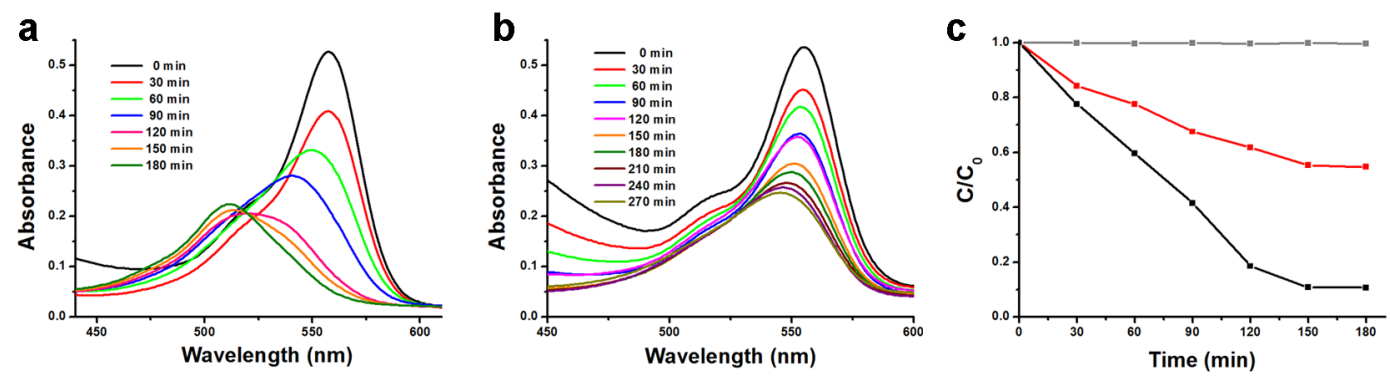
Figure 4. UV-Vis spectral changes of aqueous $\mathrm{RhB}$ in the presence of (a) $\mathrm{CdS} \bullet \mathrm{PI}-b$-PAA/graphene ensemble, and (b) reference $\mathrm{CdS} \bullet \mathrm{PI}-b$-PAA, under visible light irradiation in the presence of air. (c) Photocatalytic performance of CdS•PI- $b$-PAA/graphene (black), reference CdS $\bullet$ PI- $b$-PAA (red) and exfoliated graphene (grey) in the degradation of aqueous $\mathrm{RhB}$ under visible light irradiation in the presence of air.

\subsection{Mechanism for the photocatalytic activity of $C d S \bullet P I-b-P A A / g r a p h e n e$}

Based on the photoluminescence quenching of photoexcited CdS in CdS•PI- $b$-PAA/graphene (cf. Figure 2 (b)) that is mainly benefitted by electron-transfer phenomena, it is reasonable to state that effective charge-separation within CdS•PI- $b$-PAA/graphene ensemble is the fundamental process that dictates both the photoreduction of 4-nitroaniline and the photodegradation of $\mathrm{RhB}$ under the particular aforementioned experimental conditions. The presence of exfoliated graphene in CdS•PI- $b$-PAA/graphene ensemble allows high mobility of electrons throughout the carbon conjugated network, in stark contrast with the case of defected GO and RGO in which the process is disrupted, yet with reduced charge-recombination rate of the photogenerated holes and electrons as compared with CdS•PI- $b$-PAA in which ultra-fast recombination occurs. Accumulations of electrons in exfoliated graphene results in water splitting and evolution of $\mathrm{H}_{2}$ which effects the reduction of 4-nitroaniline to benzene-1,4-diamine (Figure 5). The photocatalytic activity of CdS•PI- $b$-PAA/graphene toward the production of $\mathrm{H}_{2}$ is particularly efficient in the presence of ammonium formate, as sacrificial electron-donor that quenches the photogenerated holes, thus promptly restoring the semiconductor CdS. On the other hand and in the presence of molecular oxygen, the accumulated electrons on exfoliated graphene are able to generate superoxide radical anions $\mathrm{O}_{2} \bullet^{-}$which in turn yield $\mathrm{HO} \bullet$ and $\mathrm{HOO} \bullet$ as highly reactive species responsible for degrading $\mathrm{RhB}$, as well as other organic pollutants [31]. In addition, the photogenerated holes on CdS similarly degrade RhB (Figure 5). 


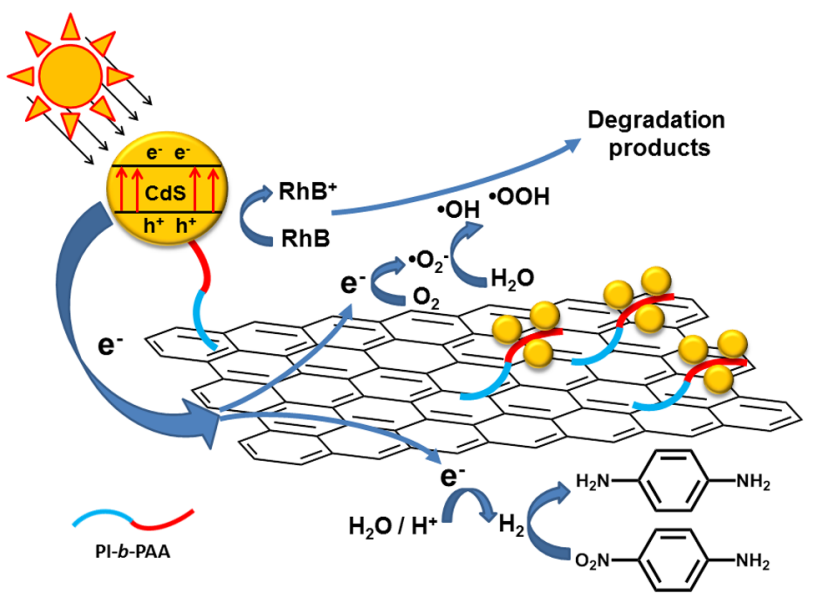

Figure 5.Illustration of the proposed mechanism for the photocatalytic processes responsible for the water splitting and $\mathrm{H}_{2}$ production (under nitrogen) and the degradation of aqueous $\mathrm{RhB}$ (under air) by visible light irradiation of $\mathrm{CdS} \bullet \mathrm{PI}-b$-PAA/graphene ensemble.

\section{Conclusions}

In summary, aqueous dispersions of CdS•PI- $b$-PAA/graphene ensembles were prepared. The CdS nanoparticles decorating the exfoliated graphene in CdS•PI- $b$-PAA/graphene were found to possess spherical morphology with diameter 3-5 nm as identified by HR-TEM and EDX spectroscopy. Furthermore, the CdS•PI- $b$-PAA/graphene was characterized by UV-Vis and photoluminescence spectroscopy. The latter aided to identify electronic communication between CdS and exfoliated graphene, within $\mathrm{CdS} \bullet \mathrm{PI}-b$-PAA/graphene, in the excited state due to significant quenching of the characteristic emission of CdS at $649 \mathrm{~nm}$, upon photoexcitation at $400 \mathrm{~nm}$, in the presence of exfoliated graphene. Then, the photocatalytic application of CdS•PI- $b$-PAA/graphene for the production of hydrogen, by examining the reduction of 4-nitroaniline to benzene-1,4-diamine, was assessed. Monitoring temporal changes in the absorption spectrum of 4-nitroaniline under visible light irradiation, it was found that the formation of benzene-1,4-diamine was faster and more efficient in the presence of CdS•PI- $b$ PAA/graphene ( $>80 \pm 4 \%$ at $20 \mathrm{~min} ; 100 \%$ at $24 \mathrm{~min}$ ) as compared when reference CdS•PI- $b$-PAA was used as photocatalyst $(<30 \pm 3 \%$ at $20 \mathrm{~min} ; 100 \%$ at $240 \mathrm{~min})$. In addition, the photocatalytic degradation of $\mathrm{RhB}$ was investigated by $\mathrm{CdS} \bullet \mathrm{PI}-b$-PAA/graphene and found to proceed with fast kinetics under visible light illumination in the presence of air. Both photocatalytic processes tested utilizing CdS•PI- $b$ PAA/graphene, involved visible light absorption by $\mathrm{CdS}$, photogeneration and separation of charges, migration of holes to $\mathrm{CdS}$ and electrons to exfoliated graphene, were superior in efficiency as compared 
either with reference CdS•PI- $b$-PAA or only exfoliated graphene, highlighting the decisive role of exfoliated graphene when participating in donor-acceptor systems and electron-transfer reactions.

\section{Acknowledgements}

Partial financial support from the Greek General Secretariat for Research and Technology and the European Commission, through the European Social Fund for Regional Development, NSRF 2007-2013

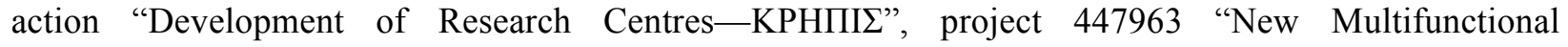
Nanostructured Materials and Devices-POLYNANO" as well as action "ARISTEIA II", project FUNGRAPH (3150) "Functionalization of graphene with multichromophoric arrays of photoactive units for energy conversion" is acknowledged. We are deeply indebted to Prof. Hisanori Shinohara and his group for kindly providing us access to HR-TEM and EDX facilities at the Chemistry Department, Nagoya University, Japan.

\section{References}

[1]. Katsukis G, Malig J, Schulz-Drost C, Leubner S, Jux N and Guldi D M 2012 Toward Combining Graphene and QDs: Assembling CdTe QDs to exfoliated graphite and nanographene in water ACS Nano 6 1915-24

[2]. Malig J, Romero-Nieto C, Jux N and Guldi D M 2012 Integrating water-soluble graphene into porphyrin nanohybrids Adv. Mater. 24 (6) 800-5

[3]. Xiang Q, Yu J and Jaroniec M 2012 Graphene-based semiconductor photocatalysts Chem. Soc. Rev. $41782-96$

[4]. Zhang N, Zhang Y and Xu Y-J 2012 Recent progress on graphene-based photocatalysts: current status and future perspectives Nanoscale 4 5792-813

[5]. Han L, Wang P and Dong S 2012 Progress in graphene-based photoactive nanocomposites as a promising class of photocatalyst Nanoscale 4 5814-25

[6]. An X and Yu J C 2011 Graphene-based photocatalytic composites RSC Advances 1 1426-34

[7]. Guo C X, Yang H B, Sheng Z M, Lu Z S, Song Q L and Li C M 2010 Layered Graphene/quantum dots for photovoltaic devices Angew. Chem., Int. Ed. 49 3014-17 
[8]. Wang Y, Yao H-B, Wang X-H and Yu S-H 2011 One-pot facile decoration of CdSe quantum dots on graphene nanosheets: novel graphene-CdSe nanocomposites with tunable fluorescent properties $J$. Mater. Chem. 21 562-6

[9]. Jia L, Wang D-H, Huang Y-X, Xu A-W and Yu H-Q 2011 Highly durable N-doped graphene/CdS nanocomposites with enhanced photocatalytic hydrogen evolution from water under visible light irradiation J. Phys. Chem. C $11511466-73$

[10]. Zhang N, Zhang Y, Pan X, Fu X, Liu S and Xu Y-J 2011 Assembly of CdS nanoparticles on the two-dimensional graphene scaffold as visible-light-driven photocatalyst for selective organic transformation under ambient conditions J. Phys. Chem. C 115 23501-11

[11]. Ye A, Fan W, Zhang Q, Deng W and Wang Y 2012 CdS-graphene and CdS-CNT nanocomposites as visible-light photocatalysts for hydrogen evolution and organic dye degradation Catal Sci Tech $\mathbf{2}$ 969-78

[12]. Latorre-Sánchez M, Primo A and García H 2013 P-doped graphene obtained by pyrolysis of modified alginate as a photocatalyst for hydrogen generation from water-methanol mixtures Angew. Chem., Int. Ed. 52 11813-6

[13]. Fang Z, Wang Y, Song J, Sun Y, Zhou J, Xu R and Duan H 2013 Immobilizing CdS quantum dots and dendritic Pt nanocrystals on thiolated graphene nanosheets toward highly efficient photocatalytic $\mathrm{H}_{2}$ evolution Nanoscale 5 9830-8

[14]. Pan D, Xi C, Li Z, Wang L, Chen Z, Lu B and Wu M 2013 Electrophoretic fabrication of highly robust, efficient, and benign heterojunction photoelectrocatalysts based on graphene-quantum-dot sensitized $\mathrm{TiO}_{2}$ nanotube arrays J. Mater. Chem. A $13551-5$

[15]. Lotya M, Hernandez Y, King P J, Smith R J, Nicolosi V, Karlsson L S, Blighe F M, De S, Wang Z, McGovern I T, Duesberg G S and Coleman J N 2009 Liquid phase production of graphene by exfoliation of graphite in surfactant/water solutions J. Am. Chem. Soc. 131 3611-20

[16]. Khan U, Porwal H, O’Neill A, Nawaz K, May P and Coleman J N 2011 Solvent-Exfoliated Graphene at extremely high concentration Langmuir 27 9077-82

[17]. Coleman J N 2012 Liquid exfoliation of defect-free graphene Acc. Chem. Res. 46 14-22 
[18]. Cook T R, Dogutan D K, Reece S Y, Surendranath Y, Teets T S and Nocera D G 2010 Solar energy supply and storage for the legacy and nonlegacy worlds Chem. Rev. (Washington, DC, U. S.)110 6474-502

[19]. Barber J 2009 Photosynthetic energy conversion: natural and artificial Chem. Soc. Rev. 38185

[20]. Forgacs E, Cserháti T and Oros G 2004 Removal of synthetic dyes from wastewaters: a review. Environ. Int. 30 953-71

[21]. Ge L, Xu M, Fang H, Photo-catalytic degradation of methyl orange and formaldehyde by $\mathrm{Ag} / \mathrm{InVO}_{4}-\mathrm{TiO}_{2}$ thin films under visible-light irradiation 2006 J. Mol. Catal. A: Chem. 258 68-76

[22]. Zhang Z, Zuo F and Feng P 2010 Hard template synthesis of crystalline mesoporous anatase $\mathrm{TiO}_{2}$ for photocatalytic hydrogen evolution J. Mater. Chem. 20 2206-12

[23]. Lai Y, Meng M, Yu Y, Wang X and Ding T 2011 Photoluminescence and photocatalysis of the flower-like nano-ZnO photocatalysts prepared by a facile hydrothermal method with or without ultrasonic assistance Appl. Catal. B 105 335-45

[24]. Zhao J, Wu T, Wu K, Oikawa K, Hidaka Hand Serpone N 1998 Photoassisted degradation of dye pollutants. 3. Degradation of the cationic dye rhodamine $B$ in aqueous anionic surfactant $/ \mathrm{TiO}_{2}$ dispersions under visible light irradiation: evidence for the need of substrate adsorption on $\mathrm{TiO}_{2}$ particles Environ. Sci. Technol. 32 2394-400

[25]. Yu K, Yang S, He H, Sun C, Gu C and Ju Y 2009 Visible light-driven photocatalytic degradation of rhodamine $\mathrm{B}$ over $\mathrm{NaBiO}_{3}$ : pathways and mechanism J. Phys. Chem. A 113 10024-32

[26]. Hu, X, Mohamood T, Ma W, Chen C and Zhao J 2006 Oxidative decomposition of rhodamine B dye in the presence of $\mathrm{VO}_{2}{ }^{+}$and/or $\mathrm{Pt}(\mathrm{IV})$ under visible light irradiation: $\mathrm{N}$-deethylation, chromophore cleavage, and mineralization J. Phys. Chem. B $11026012-8$

[27]. Skaltsas T, Karousis N, Yan H-J, Wang C-R, Pispas S and Tagmatarchis N 2012 Graphene exfoliation in organic solvents and switching solubility in aqueous media with the aid of amphiphilic block copolymers. J. Mater. Chem. 22 21507-12

[28]. Pispas S 2006 Soluble complexes of sodium poly(isoprene-b-methacrylate) micelles with cationic surfactants in aqueous media J. Phys. Chem. B $1102649-55$ 
[29]. Skaltsas T, Pispas S and Tagmatarchis N 2013 Photoinduced charge-transfer interactions on a graphene/block copolymer electrostatically bound to tetracationic porphyrin in aqueous media Chem.-Eur.J. 19 9286-90

[30]. Watanabe T, Takizawa T and Honda K 1977 Photocatalysis through excitation of adsorbates. 1. Highly efficient N-deethylation of rhodamine B adsorbed to cadmium sulfide J. Phys. Chem. 81 $1845-51$

[31]. Hoffmann M R, Martin S T, Choi W and Bahnemann D W 1995 Environmental applications of semiconductor photocatalysis Chem. Rev. (Washington, DC, U. S.) 95 69-96 